muscle involvement (ASAT normal value), IL-6 normal range. Exclusion criteria: subjects aged more than 7 years diagnosed with bacterial LRTI.

Results: Among 88 included children, 68 subjects had viral etiology and 20 children had bacterial cause (based on IL-6 low level). The LDH mean value in viral LRTI was higher than bacterial LDH mean values, not only for D0, but also for D4, without statistical correlation. There is no significant correlation between LDH mean value in D0 and D4 in viral LRTI ( $p$ value 0,150 ). The authors didn't find correlations between LDH and CRP mean values or between IL-6 and CRP.

Conclusions: LDH isn't a sensitive marker for etiological diagnosis of LRTI.

1310

\section{RESPIRATORY SYNCYTIAL VIRUS BRONCHIOLITIS IN CHILDREN WITH ACUTE LUNG INJURY IS ASSOCIATED WITH A HIGH INCIDENCE OF CONCOMITANT BACTERIAL PNEUMONIA}

L. van Helmond, M.M. IJland, C. Neeleman

Department of Intensive Care Medicine, Radboud University Nijmegen Medical Centre, Nijmegen, The Netherlands

Objective: Concomitant bacterial pneumonia (CBP) in children with respiratory syncytial virus (RSV) bronchiolitis is considered to be low and the use of antibiotics is generally discouraged. Our experience however suggested that this incidence is higher in children with RSV and acute lung injury (ALI).Our objective was to determine the incidence of CBP in children mechanically ventilated for RSV and ALI.

Methods: We conducted a retrospective chart review of all patients with $\mathrm{RSV}$ and $\mathrm{ALI}$ requiring mechanical ventilation and admitted to the PICU of a tertiary care medical center from 2004 - 2009. All patients were identified in the PICU database and were screened for ALI. Composite diagnosis of CBP was made using 4 criteria:

1) Trachea aspirate cultures at/near the time of intubation,

2) Chest radiograph,

3) Blood culture,

4) WBC abnormality.
Results: A total of 95 patients were included. Fiftyfive $(57.9 \%)$ were diagnosed as having CBP; 26 $(27.4 \%)$ met two criteria (possible CBP) and 29 $(30.5 \%)>2$ criteria (probable CPB). In infants with CBP a lower oxygenation index, a longer length of stay and less ventilator free days were observed. All children with CBP and $97 \%$ of patients without CPB received antibiotics.

Conclusion: A high incidence of CBP was observed in children with RSV and ALI requiring mechanical ventilation. The use of empiric antibiotics for pending culture results may be justified in this specific patient group.

\section{1}

\section{RESPIRATORY SYNDROME AT THE ONSET OF IDIOPATHIC PULMONARY HEMOSIDEROSIS IN CHILDREN}

\section{E.C. Bica, R. Nedelcuta, R. Diaconu, D. Bulucea \\ Pediatrics, Municipal Hospital Filantropia, Craiova, Romania}

Background: Idiopathic pulmonary hemosiderosis $(\mathrm{IPH})$ is a rare, chronic pulmonary disease with etiology still unknown and a dual symptomatology, sanguine and respiratory, that is evolving especially alternative and as a result many error of interpretation can appear.

Methods: We conducted a retrospective and prospective study, collecting cases of IPH from Romanian Pediatric Clinics, diagnosed between 1958 and 2009.

Results: We found 42 cases diagnosed with $\mathrm{IPH}$, but only 4 cases presented predominantly respiratory syndrome at the onset $(9,5 \%), 3$ of those being first interpreted as primary pulmonary tuberculosis and treated as such. 17 cases $(40,4 \%)$ presented both syndromes at the beginning and 21 cases $(50 \%)$ presented predominantly anemic symptomatology.Dry cough and dyspnea were the principal symptoms of the functional respiratory syndrome in our cases (13 cases, respectively, 12 cases). Dyspnea was associated, more frequently, with severe anemia due to hemosiderin deposition from pulmonary hemorrhages. Haemoptysis was the principal physical respiratory sign and it was present in only 11 cases at the beginning of the disease. Its intensity varied from haemoptoic sputum until normal macroscopic sputum, with positive hemosiderin-laden macrophages at the histological exam. We also had two cases of false 
hematemesis/occult gastrointestinal hemorrhages which has increased the incidence of haemoptysis in our study at $30,95 \%$.

Conclusion: Although IPH is a pulmonary disease, the respiratory syndrome is present on a second level, anemia being the primary symptom in our cases.

1312

\section{PULMONARY INFECTIONS IN CHILDREN WITH CYSTIC FIBROSIS}

\section{D.T. Qosaj}

\section{Pulmonology Department, University Clinical Centre of Kosova, Prishtina, Albania}

Introduction: Cystic Fibrosis (CF) is the most common inherited life shortening disease among white population but also appears among other ethnic groups and races. CF is autosome recesive disease and in Europe the most frequent mutation is delta F508.Disease is polysistemic and invols in first place respiratory system,gastrointestinal system,failure to thrive and other signs.

Aim of study: Presentation of cases treated in our ward,some clinical features focusing in most frequent infections which we found in our patients. during period of 2000-2008.

Material and methods: For the tudy we used patients records, outpatients records, hematologic laboratory findings, microbiology analyses, chest $X$ ray.

Results: We treat 32 children with diagnose as CF and $19(59.3 \%)$ are female and $13(40.6 \%)$ are male. Under 1 year of age are 8 children(25\%), from 1 till 5 years of age are18 children $(56.2 \%)$, from 5 till 10 years are 4 children(12.5\%) and above 10 years 2 children(0.62\%). Our diagnose was based in clinical features,sweat test and genetic mutation. In 6 children we had a positive family history(18\%). Frequency of their admission is as follows: 10 children(31.2\%) were admitted once a year, 9 children(28.1\%) we admitted 2 or 3 times a year and 16 children $(50 \%)$ were admitted more than three times during one year. Infectons that we isolated in ourchildren is follows:at 3 children(9.3\%)was isolated Streptococcus pneumonae, at 5 children(15.6\%) Echeria coli,at 3 children (9.3\%):Klebsiella, at 4 children(12.5\%) :Staphylococcus aureus,at 2 children(6.2\%) :Pseudomonas aeruginosa, at 2 children(6.2\%):Citrobacter,1 child(3.1\%) had
Branhamella.At other children the culture was sterile.

Conclusion: Presented cases realize that the infection is the common reason for exacerbation and admission in hospital. The agents are common as we found in literature.

Keywords: Cystic fibrosis,infection

1313

\section{ENDOTRACHEAL TUBE SIZE AND PLACEMENT IN NEONATES}

\author{
J.E. Tan'1, A. Foran², S. Ryan ${ }^{3}$ \\ ${ }^{1}$ Paediatrics-Neonates, ${ }^{2}$ Neonatology, ${ }^{3}$ Radiology, \\ Rotunda Maternity Hospital, Dublin, Ireland
}

Introduction: In neonates, accurate endotracheal tube (ET) size and placement is essential for adequate ventilation and surfactant delivery. Malposition of ET may cause asymmetrical surfactant distribution,pneumothorax, tracheal oesophageal perforation and aspiration.

Objectives: To assess: 1)ET placement 2) knowledge of correct ET placement and size in neonates.

\section{Methods:}

1. Chest X-rays (CXRs) of intubated infants were reviewed and verified by consultant radiologist JanMar 2010.

2. Questionnaires were distributed to consultants, NCHDs and NICU nurses in Rotunda and nationally during a week of duty in National Neonatal Transport Service. When 2 answers were selected, both smaller ET size and shorter ET length was chosen.

\section{Results:}

1. In total 203 CXRs were reviewed. $70 \%$ showed ETs were placed in the upper and middle $3^{\text {rd }}$ of the trachea. Only 4\% ETs were placed in the right main bronchus. No major complications noted.

2. In total 51 questionnaires were completed, 31 from tertiary unit and 20 from peripheral. From tertiary hospital, all consultants answered correctly, 10/13 NCHDs and 8/14 NICU nurses answered correctly. From the peripheral hospitals, all consultants answered correctly, 6/15 NCHDs and $1 / 3$ NIU nurses answered correctly. The variance 